International Journal of Modern Physics A

(C) World Scientific Publishing Company

\title{
PROBING NUCLEON STRUCTURE WITH MESON ELECTRO-PRODUCTION IN HALL C.
}

\author{
Stephen A. Wood \\ Thomas Jefferson National Accelerator Facility, \\ Newport News, Virginia 23602, U.S.A. \\ saw@jlab.org
}

\begin{abstract}
Meson electro-production is used in Hall $\mathrm{C}$ at Jefferson Lab to probe nucleon, baryon and nuclear structure. The experimental program in Hall $\mathrm{C}$ includes studies of semi-inclusive pion production, $p, d\left(e, e^{\prime} \pi^{ \pm}\right) X$, where low energy factorization has been observed, suggesting that these reactions can be used to probe nucleon structure, including transverse momentum distributions of quarks, at energies available at JLab after the upcoming 12 GeV upgrade.

Keywords: JLab; SIDIS; QCD; Meson Production

PACS numbers: 13.60.Hb, 13.60.Le.
\end{abstract}

\section{Hall $\mathrm{C}$ at Jefferson Lab}

Hall C, one of three endstations at the Thomas Jefferson National Accelerator Facility (JLab), hosts a variety of particle and nuclear physics experiments, many of which involve meson electro-production.

The current base equipment in Hall $\mathrm{C}$ includes two magnetic spectrometers, the 7.5 GeV/c High Momentum Spectrometer (HMS) and the $1.8 \mathrm{GeV} / \mathrm{c}$ Short Orbit Spectrometer (SOS). These spectrometers, with momentum acceptances of $18 \%$ and $40 \%$ respectively, accept solid angles of over $6 \mathrm{msr}$. With the detector packages in the focal plane huts, these focusing magnetic spectrometers have momentum resolutions better than $1 \times 10^{-3}$, angular resolutions less than $1 \mathrm{mr}$ and have good particle ID capabilities to separate electrons, pions, kaons and protons. The detector packages are well shielded allowing these spectrometers to operate with high luminosities and measure small cross sections.

An upgrade at JLab is underway that will increase the accelerator energy, providing $12 \mathrm{GeV}$ to a new endstation, and up to $11 \mathrm{GeV}$ to the existing endstations, including Hall C. As part of this upgrade, Hall C is building a new magnetic spectrometer, the Super High Momentum Spectrometer (SHMS) which will replace the SOS. The SHMS, will be comprised of five superconducting magnets and can be set to central momenta from 2 to $11 \mathrm{GeV} / \mathrm{c}$. The first magnet on the SHMS will be a horizontal bender which will kick scattered particles to a larger angle, allowing the SHMS to be set to central scattering angles as low as $5.5^{\circ}$. With resolutions and 


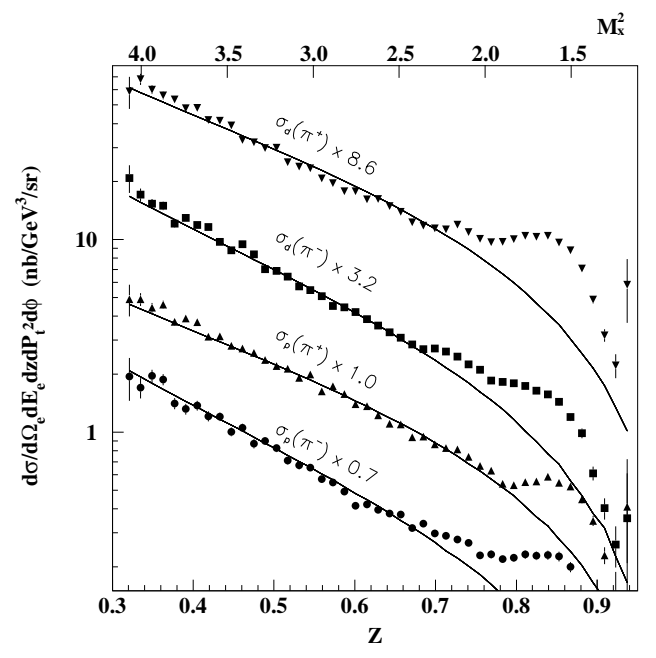

Fig. 1. $p\left(e, e^{\prime} \pi^{ \pm}\right) X$ and $d\left(e, e^{\prime} \pi^{ \pm}\right) X$ cross sections at $x=0.32$ compared to a prediction assuming factorization.

particle ID capabilities similar to the HMS, Hall C's ability to measure low cross sections and make precision Rosenbluth separations will be maintained.

\section{Semi-inclusive Deep Inelastic Scattering}

The semi-inclusive process $e N \rightarrow e \pi^{ \pm} X$ has been shown, in the high energy limit, to factorize into a hard part describing the interaction of the virtual photon with a quark and a soft part describing the hadronization of the quark (Fragmentation functions) $)^{1}$. This factorization can be written as $\sigma_{\text {SIDIS }} \sim$ $\sum_{q} e_{q}^{2} q\left(x, Q^{2}\right) D_{q \rightarrow \pi}\left(z, Q^{2}\right) b e^{-b P_{t}^{2}}$, where $z=E_{\pi} /\left(E_{e}-E_{e^{\prime}}\right)$ is the fraction of the available energy carried by the detected pion and $P_{t}$ is transverse momentum of the pion relative to the momentum transfer direction defined by the scattering electron. If factorization of this process, known as Semi-Inclusive Deep Inelastic Scattering (SIDIS) holds at JLab accessible kinematics, then this reaction holds the potential of flavor tagging quark distributions and studying quark transverse momentum distributions by taking advantage of the higher luminosities, larger cross sections, and spectrometer properties available at JLab.

Exploratory measurements of semi-inclusive $p\left(e, e^{\prime} \pi^{ \pm}\right) X$ and $d\left(e, e^{\prime} \pi^{ \pm}\right) X$ were made in Hall $\mathrm{C}^{2}$ with electrons detected by the SOS and pions by the HMS at a photon virtuality of $Q^{2}=2.3 \mathrm{GeV}^{2}$ with a central kinematics of $x=0.32$ and $z=0.55$. About these central kinematics, three scans were taken, measuring cross section dependencies on $z(0.3-1.0)$, Bjorken $x(0.25-0.6)$ and $\theta_{\pi}\left(0^{\circ}-8^{\circ}\right)$. In figure 1 , the $z$ scan cross sections are compared to a high energy prediction assuming factorization, using CTEQ5 parton distributions ${ }^{3}$ and fragmentation functions from Binnewies et al. ${ }^{4}$. For $z>0.7$, the data rise above the prediction, peaking at a $z$ 

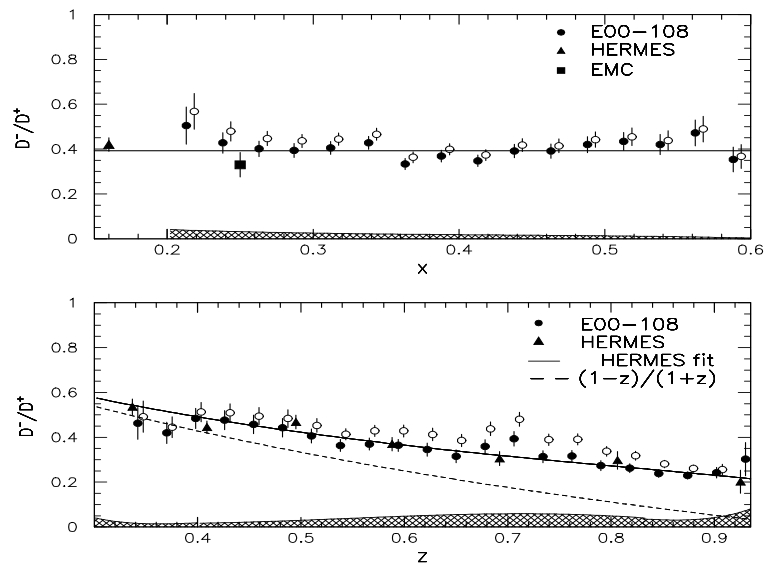

Fig. 2. Dependence of un-favored to favored fragmentation function ratios on $x$ and $z$.

that corresponds to the $\Delta(1232)$ mass in the unobserved reaction products. Below 0.7 the data match the predictions well, suggesting that factorization is valid, at least numerically, at these low energies. Furthermore, ratios of the cross sections, behave close to simple quark-parton model expectations. Using the above factorization expression, the ratio of $\pi^{+}$to $\pi^{-}$production can be used to derive the ratio of favored $\left(D^{+}\right)$to un-favored $\left(D^{-}\right)$fragmentation functions. (The un-favored fragmentation function describes hadronization into a pion that does not contain the flavor of the struck quark.) The fragmentation function ratio varies smoothly with $z$ even through the $\Delta$ region and over $0.2<x<0.6$ the ratio is flat as expected from the lack of $x$ dependence in the fragmentations functions. (Fig. 2)

In the factorization model of SIDIS, the transverse momentum $P_{t}$ of mesons arises from the combination of the transverse momentum of the struck parton $\left(k_{t}\right)$ and the transverse momentum acquired in the fragmentation of the struck quark $\left(z k_{t}\right), P_{t}=z k_{t}+p_{t}$. By fitting the $P_{t}$ distributions from the four $p, d\left(e, e^{\prime} \pi^{ \pm}\right) X$ measurements, using a gaussian shape for the distributions, and by making several assumptions, widths can be extracted for the transverse momentum distributions of the quarks and the fragmentation functions ${ }^{5}$. This fit (Fig. 3) gives similar widths for the favored and un-favored fragmentation functions and a larger width for $d$ quarks than $u$ quarks, as suggested by a di-quark model of the proton.

\subsection{Planned 12 GeV SIDIS experiments}

A large program to explore SIDIS will be carried out at JLab after the $12 \mathrm{GeV}$ upgrade. Several of the initial experiments will use the good angular and momentum resolutions of the SHMS/HMS spectrometer pair, and set the basis for using SIDIS at JLab energies as a tool for flavor tagging of quark distributions. E12-06-104 ${ }^{6}$ will perform Rosenbluth separations to measure the ratio of longitudinal to transverse 

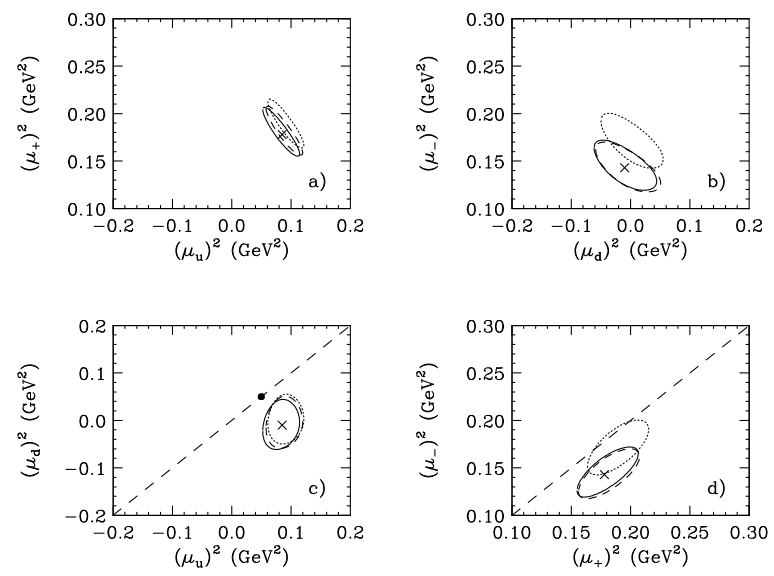

Fig. 3. Crosses are parameters from a fit to transverse pion momentum distributions in the $p, e\left(e, e^{\prime} \pi^{ \pm}\right) X$ reactions and the ellipses are standard deviation contours with different diffractive $\rho$ production and radiative background subtraction assumptions. $u_{u}^{2}$ and $u_{d}^{2}$ are quark widths squared, and $u_{+}^{2}$ and $u_{-}^{2}$ are the widths squared for favored and unfavored fragmentation functions.

cross sections in pion electroproduction. The ratio $R=\sigma_{L} / \sigma_{T}$ is well measured in inclusive electron scattering (DIS). It has been assumed that $R$ for semi-inclusive pion production behaves the same as for DIS, but this assumption has not been checked. This experiment will map out SIDIS R for a variety of kinematics and examine the behavior in the exclusive limit $(z \rightarrow 1)$. A second experiment, E12-09$017^{7}$ will extend measurements of the transverse momentum dependence of pions in SIDIS, constraining the transverse widths of $u / d$ quarks and fragmentation functions.

An interesting application of SIDIS is to measure or set limits on charge symmetry in valence quark distributions. Charge symmetry, the assumption that $u^{p}(x)=d^{n}(x)$ and $d^{p}(x)=u^{n}(x)$, which is usually assumed in quark distributions but has not been well tested, would have interesting implications if strongly violated. Experiment E12-09-002 ${ }^{8}$ plans to measure the ratio of $d\left(e, e^{\prime} \pi^{+}\right) X$ to $d\left(e, e^{\prime} \pi^{-}\right) X$ cross sections over a range of $Q^{2}, x$ and $z$. By careful control of the relative detection efficiency of $\pi^{+}$vs. $\pi^{-}$, this ratio, which can be related to charge symmetry violation (CSV) of quark distributions, can be measured to a precision of $1 \%$. The measurement will substantially improve the limits on CSV set by MRST group studies $^{9}$ of parton distribution uncertainties (Fig. 4).

\section{Other applications of meson production in Hall $\mathrm{C}$}

The $Q^{2}$ behavior of the exclusive $p\left(e, e^{\prime} \pi^{+}\right) n$ reaction has been studied, showing hints of a $Q^{2}$ scaling of the longitudinal cross section consistent with QCD factorization ${ }^{10}$. As a test of the validity of extracting Generalized Parton Distributions at JLab energies, a future experiment will test this scaling and the presumed 


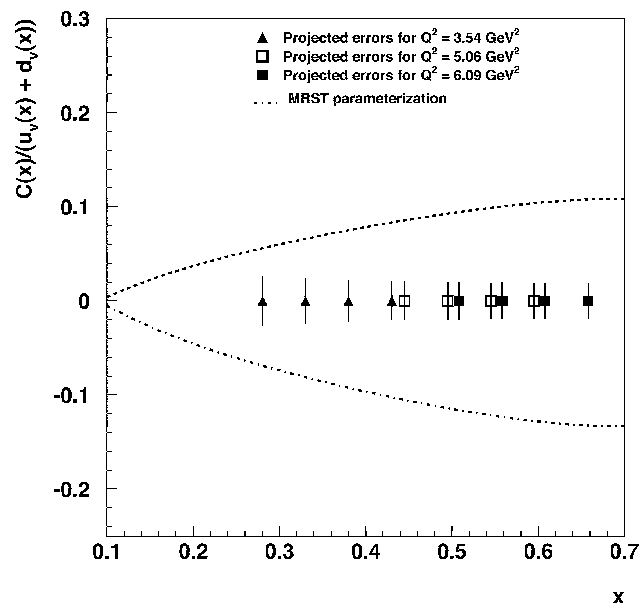

Fig. 4. Projected errors on valence quark charge symmetry violation, $C(x)=\left(u_{v}^{p}(x)-d_{v}^{n}(x)\right)-$ $\left(d_{v}^{p}(x)-u_{v}^{n}(x)\right)$, compared to limits set by MRST.

dominance of $\sigma_{L}$ over $\sigma_{T}$ with a larger $Q^{2}$ range $^{11}$. These scaling studies will be extended to exclusive $K^{+}$electroproduction which may yield a determination of the Kaon form factor up to $Q^{2}=3 \mathrm{GeV}^{212}$.

In addition to inclusive and exclusive meson production experiments that probe nucleon structure, the Hall $\mathrm{C}$ facilities are used for a variety of experiments involving meson production. With $A\left(e, e^{\prime} \pi^{+}\right)$, the onset of color transparency, an increase in the transparency of the nucleus with increasing $Q^{2}$, has been observed ${ }^{13}$. This measurement, which uses the nucleus as a probe of the meson electro-production mechanism, will be continued to higher $Q^{2}$ after the upgrade. Transition amplitudes to the baryon resonances $\Delta(1232)$ and $S_{11}$ have been measured at $Q^{2}$ values about $7 \mathrm{GeV}^{2}$ with $\pi^{0}$ and $\eta$ production ${ }^{14,15}$.

$K^{+}$electro-production on nuclei, $A\left(e, e^{\prime} K^{+}\right) A_{\Lambda}$, gives access to unique hypernuclei. With the narrow energy spread of the JLab electron beam and custom electron and kaon spectrometers, hypernuclei and levels such as ${ }_{\Lambda}^{7} \mathrm{Li}$ and ${ }_{\Lambda}^{28} \mathrm{Al}$ have been observed with FWHM resolutions of $400 \mathrm{KeV}^{16}$.

\section{Acknowledgments}

The author thanks H. Mkrtchyan and the E00-108 collaboration. This work was supported by DOE contract No. DE-AC05-06OR23177, under which Jefferson Science Associates, LLC (JSA) operates the Thomas Jefferson National Accelerator Facility. 


\section{References}

\section{References}

1. X. Ji, J.-P. Ma, F. Yuan, Phys. Lett. B 597,299 (2004).

2. T. Navasardyan et al., Phys. Rev. Lett. 98, 022001 (2007).

3. H. .L. Lai et al., Eur. Phys. J. C 12, 375 (2000).

4. J. Binnewies, B. A. Kniehl, and G. Kramer, Phys. Rev. D 52, 4947 (1995).

5. H. Mkrtchyan, P.E. Bosted et al., Phys. Lett. B 665, 20 (2008).

6. "Measurement of the Ratio $R=\sigma_{L} / \sigma_{T}$ in Semi-Inclusive Deep-Inelastic Scattering," Jefferson Lab Experiment E12-06-104.

7. "Transverse Momentum Dependence of Semi-Inclusive Pion Production," Jefferson Lab Experiment E12-09-017.

8. "Precise Measurement of $\pi^{+} / \pi^{-}$Ratios in Semi-inclusive Deep Inelastic Scattering Part I:, Charge Symmetry Violating Quark Distributions," Jefferson Lab Experiment E12-09-002.

9. A. D. Martin, R. G. Roberts, W. J. Stirling and R. S. Thorne, Eur. Phys. J. C 35, 325 (2004).

10. T. Horn et al., Phys. Rev. C 78, 058201 (2008).

11. "Scaling Study of the L-T Separated Pion Electroproduction Cross Section at 11 GeV," Jefferson Lab Experiment E12-07-105.

12. "Studies of the L-T Separated Kaon Electroproduction Cross Section from 5-11 GeV," Jefferson Lab Experiment E12-09-011.

13. B. Clasie et al., Phys. Rev. Lett. 99, 242502 (2007).

14. A. N. Villano et al., Phys. Rev. C 80, 035203 (2009).

15. M. M. Dalton et al., Phys. Rev. C 80, 015205 (2009).

16. O. Hashimoto et al., Nucl. Phys. A835, 121 (2010). 